\title{
Energy scenarios for the Minas Gerais State in Brazil: an integrated modeling exercise using System Dynamics
}

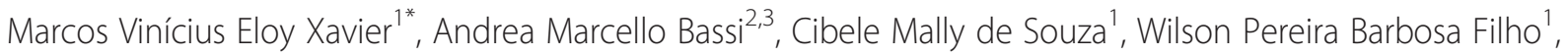
Kevin Schleiss ${ }^{1}$ and Felipe Nunes ${ }^{1}$

\begin{abstract}
Background: Developing medium- to long-term energy scenarios is a key to achieve goals of energy security and greenhouse gas emission reduction through the implementation of effective public policies.

Methods: Despite the availability of many tools and methods that focus on energy planning from an engineering perspective, only a few approaches have the methodological capabilities to build energy scenarios and quantify their impacts across social and environment indicators in a dynamic and flexible way. To fill this gap, we have created a System Dynamics model customized for the state of Minas Gerais in Brazil to simulate possible trajectories of energy demand and supply.

Results: Two scenarios were developed: a business-as-usual case (BAU), with no major changes in the energy profile of the state; the other is an alternative scenario, where the reduction of carbon intensity (RIC) is a key goal. To summarize the results, the scenario RIC has lower greenhouse gas emissions and greater energy generation and employment, but has a total cumulative cost higher than BAU as the final indicators estimated.

Conclusions: We can see important opportunities to contribute to global climate change mitigation while fostering a low-carbon energy sector and a green economy in the state. By investing in the renewable energy sources, energy efficiency, technological improvements, and the targets analyzed in this study, Minas Gerais State could reduce political and financial risks originating from the reliance on fossil fuels while generating more income in the energy sector and the state economy as a whole and reducing medium- and longer-term costs.
\end{abstract}

Keywords: Scenarios; Energy; Minas Gerais

\section{Background}

The energy sector is responsible for nearly $80 \%$ of the anthropogenic greenhouse gas emissions (GHG) globally, considering the entire energy supply chain: production, conversion, transportation, and consumption [1]. This occurs due to the high consumption of fossil fuels, which accounts for about $81 \%$ of the world energy mix, while renewable sources account for only $13 \%$ [2].

Recent years have registered solid growth in the overall use of renewable energy, reaching $4 \%$ per year on average [3]. However, even with recent incentives for

\footnotetext{
* Correspondence: marcos.xavier@meioambiente.mg.gov.br

${ }^{1}$ Fundação Estadual do Meio Ambiente (FEAM), Rodovia Prefeito Américo

Gianetti, s/n, Belo Horizonte, Minas Gerais 31630-900, Brazil

Full list of author information is available at the end of the article
}

alternative energy sources and energy efficiency policies, the share of fossil fuels (also leading to increasing GHG emissions) tends to remain dominant in the global context characterized by economic growth and threatened national energy security, both in developed and in developing countries. Reducing GHG emissions associated with energy demand and supply without impacting the quality of life of the world population will require a major effort to both (1) diversify the energy matrix and (2) change consumption patterns [1].

When comparing the Brazilian energy matrix with other countries worldwide, we notice a clear difference in the use of renewable energy. In Brazil, the share of renewable energy sources reaches $44.7 \%$ of supply (Figure 1), a value that grew to $55 \%$ for the specific case of Minas Gerais 


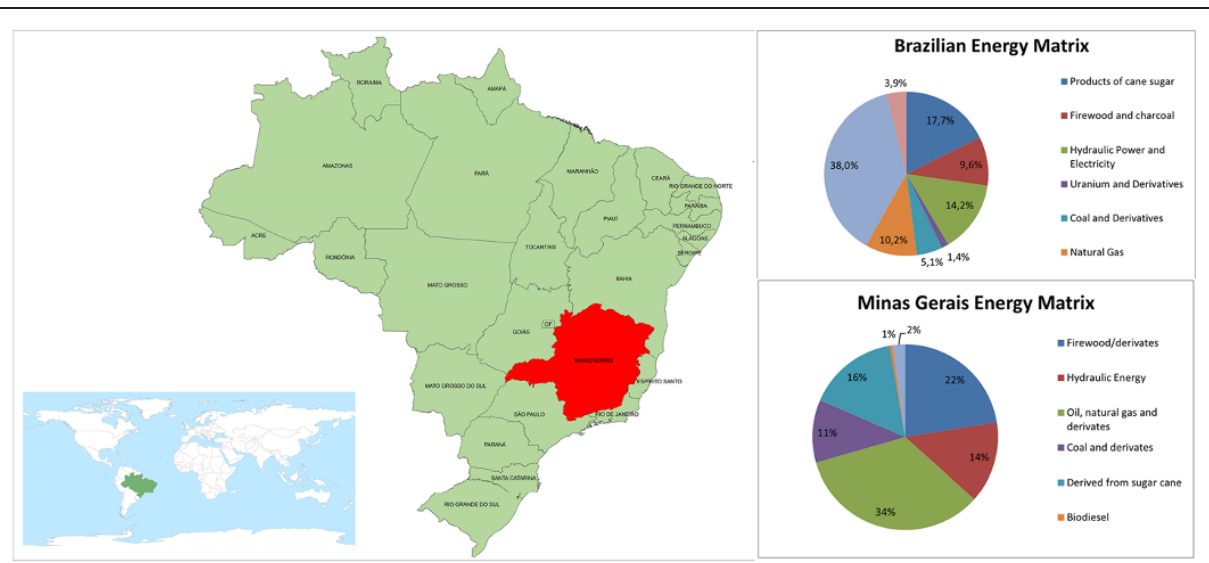

Figure 1 Brazilian and Minas Gerais state energy mix.

State [4]. Located in the southeastern region, Minas Gerais has the third largest economy and the second largest population (19.6 million) in the country. The land area represents $7 \%$ of the Brazilian territory, higher than those in countries such as France, Sweden, and Spain. The energy mix discrepancy is due to the fact that the Brazilian and Minas Gerais policies over the years have heavily promoted hydroelectricity over other options. In this context, the state of Minas Gerais plays an important role in the national scene since it produces $17 \%$ of the electricity in the whole country [4].

Regarding the GHG emissions, the energy sector in Minas Gerais (production and consumption) is the second largest, with $36.9 \%$ of the 123 million tonnes of $\mathrm{CO}_{2}$ equivalent $\left(\mathrm{CO}_{2} \mathrm{eq}\right)$, behind only Land Use, LandUse Change and Forestry sector, which accounts for roughly $51.4 \%$. Further, according to the data presented in the GHG inventory, the state emitted 6.4 tonnes of $\mathrm{CO}_{2}$ eq per capita and approximately 1.28 tonnes of $\mathrm{CO}_{2}$ eq/US\$ $1,000.00$ in 2005 [5].

Despite the low carbon intensity in the energy sector of the state, the increasing energy demand arising from population growth, rising income, and growth in industrial production, agricultural services, and gross domestic product (GDP) in general (average annual growth rate of $8.28 \%$ from 2002 to 2010 ) poses challenges to increasing, and even maintaining, the current share of renewables in the state. Moreover, some projections show the necessity to further increase energy supply in the medium term in order to effectively support the current and projected economy [4]. This is mainly due to the fact that the main power source of the state, hydroelectricity, has practically reached its maximum exploitation stage [4]. Thus, the Government faces a relevant dilemma: which strategy and energy mix should be prioritized in order to ensure simultaneously the increase in energy security and the decline in GHG emissions?
In this context, efforts should be directed towards promoting more sustainable energy generation and consumption in the state, mainly achieved by increasing the use of alternative energy sources, such as wind, solar, biomass, and even natural gas, which have lower carbon content than the other fossil fuels currently used in Minas Gerais.

Currently, the share of wind and solar energy in the energy mix of the state is almost negligible, despite the high potential. The average wind capacity at $100-\mathrm{m}$ height is estimated at approximately 39.000 MW, which represents a potential of roughly 120 million MWh and two times the energy demand in the state in 2009 (assuming an average capacity factor of 0.35) [6].

Besides the need to increase the share of these renewables sources, there is also a significant opportunity for replacing more polluting and GHG-intensive fuels, such as coal and oil fuel, with charcoal from planted (and sustainably managed) forests and natural gas in the industrial sector, and also increasing the share of biofuels in the transportation sector.

In view of the current context and upcoming challenges and opportunities, it is crucial to elaborate detailed scenarios for energy supply and demand to assist in decision-making, considering policy options for promoting renewable energy sources, fuel switching to less carbon-intensive fossil fuels, and for promoting energy efficiency. However, it is extremely important to use a methodology that allows addressing quantitatively also the potential cross-sectoral impacts of such interventions, measuring possible economic, environment, and social main impacts directly or indirectly influenced by the investments simulated.

In this context, this study aims to create energy supply and demand projections, as well as their main impacts on environmental and socioeconomic indicators, for two main scenarios: a business-as-usual (BAU) case 
and an alternative scenario in which carbon intensity would be reduced (RIC) in the state of Minas Gerais. We also intend to propose a methodology for developing scenarios that allow to identify possible future trajectories disaggregated by the economy sector, calculating specific energy consumption, estimating future GHG emissions, and creating a theoretical and methodological basis for an ex ante assessments of the cost of implementation of the public policies considered.

\section{Review of relevant modeling frameworks}

A large number of models are available for either analysis of energy or integrated national planning. Unfortunately, only few of them encompass both aspects in a single holistic framework, which would apply well to the rapidly changing context of Brazil and Minas Gerais. Feedbacks across the economy, society, and environment are difficult to identify, manage, and quantify, especially with conventional methodologies and models. Two categories of energy-economy models are commonly accepted: market- and behavior-oriented, which are both causal-descriptive (e.g., System Dynamics) or correlational (e.g., econometrics), and bottomup optimization models [7].

Policy optimization models are generally built to find the optimal intervention that minimizes expected energy supply costs at any point in time, given a specific set of assumptions and constraints. Correlational models provide projections on the implementation of policies describing the system using correlation and are based on established economic theory. System Dynamics models, instead, provide information on the functioning of the systems to analyze the wider impacts of each policy being tested [8]. These policy proposals are taken as given to support the formulation of final drafts and evaluate their impacts on society, economy and the environment, without imposing rational behavior or economic equilibrium.

System Dynamics models, thus, have more freedom to represent phenomena that are inconsistent with some of the assumptions (i.e., economic theory) of policy optimization models, allowing a full customization of their structure through the representation of feedbacks, delays, and non-linearity [9].

Early energy models were commonly bottom-up linear programming applications (using optimization) focused strictly on the assessment of energy systems. Some of these models are still being used, despite their limited scope [10]. Some linear programming models were then further developed to include non-linear programming components that allow for the interaction of "bottom-up" technology modules with "top-down" simplified macroeconomic modules $[11,12]$. Recently, due to the need to investigate the impacts of natural disasters, as well as technology development, these tools were enhanced with stochastic programming and mixed-integer programming techniques [11]. Models like Market Allocation (MARKAL) [11,13], Model of Energy Supply Systems Alternatives and their General Environmental Impacts (MESSAGE) [14,15], WEM (World Energy Model) [16] and National Energy Modeling System [17] belong to the category of models that have evolved over time and now include econometric components and a Computable General Equilibrium model (theory-based) to take into account macro-economic conditions, on top of an optimization structure representing the energy system. These models can be, in fact, considered hybrids as they try to combine top-down and bottom-up approaches. MARKAL, in particular, which nowadays represents a family of models more than a single framework, is in fact a "partial equilibrium bottom-up energy system technology optimization model employing perfect foresight and solved using linear programming; with numerous model variants that expand the core model to allow for demand response to price (MACRO (non-linear) and Elastic Demand (MED)), uncertainty (Stochastic), endogenous technology learning (ETL), material flows and multi-region (linked) models; plus new variants under development which support multi-criteria analysis (Goal Programming), and myopic execution (SAGE for EIA IEO)" [11].

The use of medium- to longer-term energy planning models over the years has provided policy makers and planners with insights on policy impacts and energy technologies, in addition to offer projections on demand and supply, as well as prices. In some cases, energy models (e.g., correlational ones) were also able to provide some insights on the interconnections between macro-economic development and energy management, but rarely vice versa (e.g., causal descriptive models). These models, such as in the case of WEM, include six main modules: final energy demand, power generation, refinery and other transformation, fossil fuel supply, $\mathrm{CO}_{2}$ emissions, and investment [18]. Their structure is generally a systems engineering optimization construction of the energy sector, in which engineering feasibility is ensured by making energy flows consistent with model constraints on primary-energy extraction and energy conversion and transport, as well as on end-use technologies and others. These models operate under perfect foresight assumptions and optimize energy flow-given demand and an objective function. This function, also called optimization routine, selects energy carriers and transformation technologies from each of the sources to produce the least-cost solution subject to the predefined (and user-defined) constraints [11].

Each model in this category slightly differs from the others in terms of details and boundaries. MESSAGE, 
for instance, finds the optimal flow of energy from primary energy sources to useful energy demand (end-use consumption) through the simulation of various investment choices that lead to the lowest cost of all feasible energy supply mixes to meet the specifically given energy demand. In other words, given exogenous demand, MESSAGE selects the energy mix that supplies it at least cost $[19,20]$. The World Energy Model instead calculates energy demand econometrically, using data for the period from 1971 to 2004. For future assumptions, adjustments can be made to account for expected changes in structure, policy or technology using econometrics [16]. MESSAGE could only calculate demand endogenously when coupled with MACRO, a Computable General Equilibrium (CGE) model that would communicate iteratively with the energy components of MESSAGE to calculate energy prices based on the best mix of energy sources used to supply demand (e.g., demand and supply balances), which is in turn calculated using GDP and energy prices. In order to calculate demand and other macro-variables in such way, economic growth and demographics have to be indicated exogenously, in addition to technology costs, technical characteristics (e.g., conversion efficiencies), and development [16,20].

A more comprehensive model that incorporates a larger number of economic components with respect to MARKAL is General Equilibrium Model for Energy-EconomyEnvironment Interactions (GEM-E3). This model computes the equilibrium prices of goods, services, labor, and capital that simultaneously clear all markets and determines the optimum balance for energy demand/ supply and emission/abatement [21].

The GEM-E3 model includes economic frameworks used by the World Bank (national accounts and social accountability matrix) as well as projections of full inputoutput tables by country/region, employment, balance of payments, public finance and revenues, household consumption, energy use and supply, and atmospheric emissions. There is no objective function in GEM-E3: being a full CGE model, the equations underlying the structure of the model define the behavior of the actors identified with the SAM [22]. The production function of the model uses capital, labor, energy, and materials; the properties of the system such as stock and flow relationships, capital accumulation delays, and agents' expectations are considered. The main exogenous inputs to the model are population, GNP, and energy intensity.

The wider boundaries of the GEM-E3 model resemble the structure of Threshold 21 (T21) [23], a causal-descriptive model, where System Dynamics (SD) is employed and where society, economy, and environment are represented. T21 and other System Dynamics models, thanks to a flexible and versatile software application, are able to combine optimization and market-behavior frameworks into one holistic framework that represents the causal structure of the system. SD models offer a complementary approach that allows moving toward optimal energy flows while concurrently simulating the interaction of a large number of feedback loops with the major factors in the rest of the economy, society, and the environment. This provides useful insights for policy formulation and evaluation analysis. Examples of SD models applied to energy issues include the IDEAS model [24], an improved version of the FOSSIL models [25,26] built by Roger Naill, the Energy Transition Model [27], the Petroleum Life Cycle Model [28], and the Feedback-Rich Energy Economy model [29]. These models do not encompass the interactions between energy, society, economy, and environment, which constitute one of the major innovations introduced by the Threshold 21 model [30,31]. In fact, the FOSSIL, IDEAS, and Life Cycle models consider energy in isolation; Sterman's model [27] includes energy-economy interactions only, and Fiddaman's FREE model [29] focuses on economy-climate interactions (oil and gas depletions are considered as "source constraints", while climate change is a "sink constraint" on the energyeconomy system). Nevertheless, both FOSSIL and IDEAS models made important contributions, such as their use by the Department of Energy for policy planning in the 1980s.

A recent System Dynamics model used as part of an Integrated Assessment Models, IMAGE 2.2, for climate change analysis is TIMER [32,33]. TIMER is a simulation model that does not optimize scenario results over a complete modeling period on the basis of perfect foresight but simulates, instead, the year-to-year investment decisions based on a combination of bottom-up engineering information and specific rules about investment behavior, fuel substitution, and technology. The output is a rather detailed picture of how energy demand, fuel costs, and competing supply technologies could develop over time in various regions. The main exogenous inputs include GDP growth, population, technological development, and resource depletion. Differently from T21, TIMER does not account for feedbacks linking the energy sector to other ones. Though the uncertainties involved in these feedbacks may be large, the lack of interrelations between the different sectors is an important limitation that is not addressed with optimization or econometric models, which is why the author attempts at proposing a more comprehensive approach to energy issues.

With respect to the local context of Brazil, the models used to support energy planning are the Model for Analysis of Energy Demand (MAED) of the International Atomic Energy Agency and MESSAGE of the International Institute for Applies System Analysis. These models are primarily chosen for their wide use by the international community, allowing for comparisons, cooperation, and dissemination of results beyond Brazil. 
The model MAED links socio-economic drivers to energy demand, which is the main output of the model. In turn, the MESSAGE model optimizes energy supply to meet the demand for useful energy.

Acknowledging strengths and weaknesses of the various models reviewed and the peculiarities of the rapidly changing environment in Minas Gerais, this study aims at exploring the dynamics of the energy sector in the state. In this respect, the System Dynamics was the methodology chosen, primarily due to the capability to develop causal descriptive models, with a flexible and modular approach that would not require reliance on national data, being customized at the state level.

As a result, the model presented in this study integrates relevant economic and social drivers, and estimates social and environmental impacts of selected policy interventions, considering feedbacks, delays, and non-linear behavior.

\section{Methods}

The model features a modular structure, borrowing from the underlying structure of the T21 model [34] (Figure 2), where similar structural assumptions are adopted and coupled with local data to create projections for several variables across sectors (including, for instance, energy demand, and greenhouse gas emissions). The key variable outputs of each module are used as inputs for calculating variables of other modules, giving the model, as a whole, a dynamic and systemic character founded on the representation and use of several feedback loops. The model consists of 20 modules which were combined and run in the platform Simulating and Assessing Public Policies of Energy and Climate Change (SAPPE) developed in the Vensim software (Ventana Systems, Inc., Harvard). These 20 modules were divided into five categories: sectors, energy production, energy demand, energy efficiency, and indicators (see Table 1).

Using differential equations and the explicit representation of stocks and flows, the model calculates, among others, the likely energy demand, aggregated jobs generation due to the investment in different energy sources, and GHG emissions for each sector. The sectors analyzed were the residential; industrial; services; agriculture, forestry, and other land uses (AFOLU); and transport. Energy supply is modeled based on the trend curve of the technologies in the BAU scenario and using assumptions in the RIC scenario. The energy trade is also estimated by comparing the curves of supply and demand for the state of Minas Gerais both in terms of quantity, as in value. With this analysis, it was possible to provide for a net surplus (positive net trade) of electricity from 2026.

The main assumptions used to calibrate and run the model include population growth (1\% per year), $6 \%$ of GDP growth per year, and a linear growth of the car fleet, reaching approximately 8 million cars by 2030 . Regarding energy prices, we assumed $2 \%$ by 2030 [35].

For the residential sector, we used specific data on households for Minas Gerais (population and energy demand). The main input variables to the model are the growth rate of population and households in the state by 2030, and the estimated electricity demand per person. The model is also using electricity prices and their impact on demand. In the

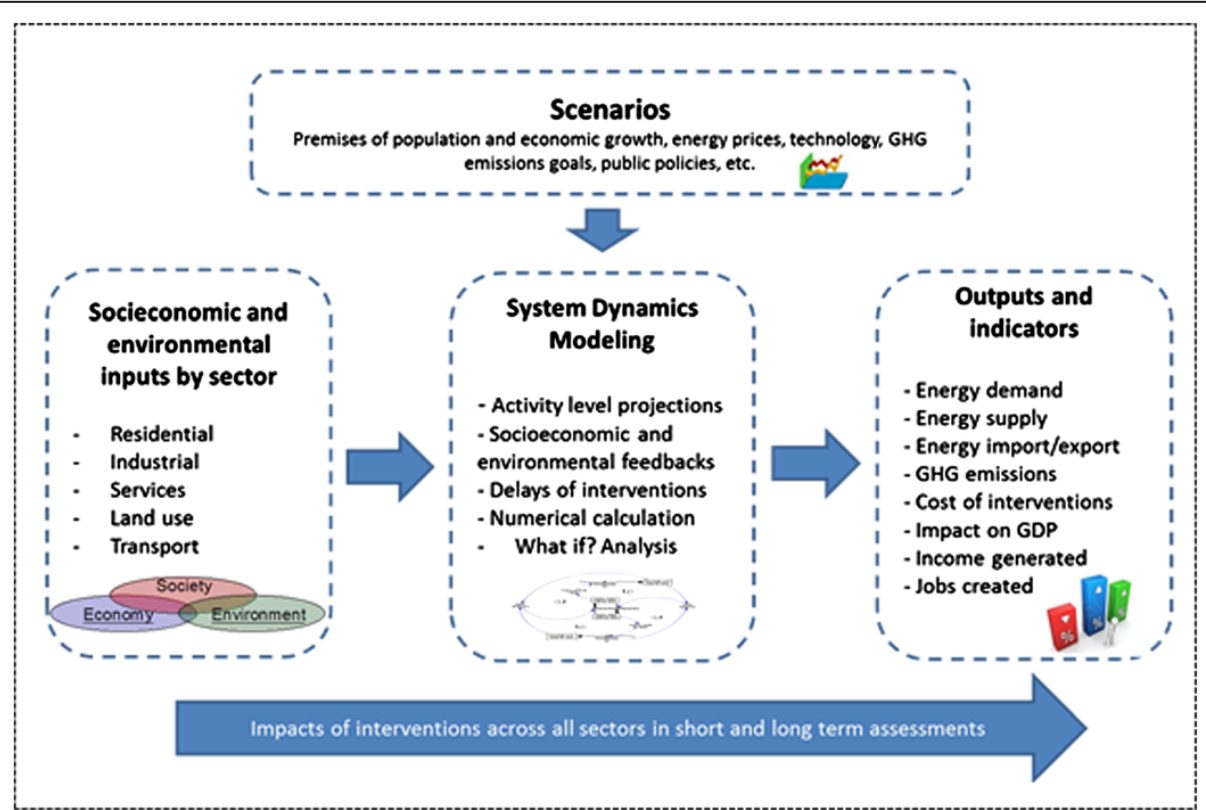

Figure 2 Schematic modeling approach. 
Table 1 The modules of the platform SAPPE

\begin{tabular}{lllll}
\hline Sectors & Energy production & Energy demand & Energy efficiency & Indicators \\
\hline Residential & Biodiesel & Electricity & Water heating & Cost of the scenario \\
Industrial & Ethanol & Oil & Lighting & Employment generated in the sectors \\
Services & Renewable electricity & Natural gas & Refrigeration & GHG emissions \\
AFOLU & Non-renewable electricity & Coal & & \\
Road transport & & Biofuels & & \\
\hline
\end{tabular}

RIC scenario, the impact of possible policies or energy efficiency measures in the residential sector was also estimated, considering the gradual disappearance of incandescent bulbs in the market; increased efficiency of refrigerators, freezers, and air conditioners; and maximized used of solar collector for heating water in general.

In the industrial sector module, we calculated the specific indicators for the state. The rationale for creating this module was based on the premise that the expected growth in Minas Gerais directly impacts industrial capital, which in turn impacts the industrial production (an elasticity factor is used to regulate this causal relation [34]). The production is also affected by investments in education and health conducted (labor productivity), and input prices such as natural gas, oil, and electricity (total factor productivity) as shown in Figure 3. The modeling of industrial production allows for calculating the energy demand, employment, and GHG emissions from the industries in the state. The elasticities used for the industrial sector include -0.15 relative to electricity price, -0.2 for oil price, -0.1 for natural gas price and 0.3 to employment.

The structure of the services sector is analogous to the industrial one. The main inputs are the expected growth in Minas Gerais by 2030 (used for calibration), the price of electricity, and the investment in education and health. These inputs are used to calculate the GDP of the services sector, also using elasticity factors as explained above. Finally, the GDP of the services sector impacts the energy demand and employment.

The sector of AFOLU contains agriculture, forestry, livestock, and changes in land use such as deforestation and reforestation. It was shaped primarily by considering three factors:

- GDP growth rate in Minas Gerais: This growth rate displays the expected growth of the economy by 2030 and determines the ability of investment in the capital and the AFOLU sector.

- Product prices in the market: The higher the price of the product, the higher the value added to the AFOLU sector.

- Income from the field: This directly affects the production sector and is influenced by several factors (water availability in the state, soil quality, soil nutrient depletion, soil erosion, among other data relating to the agricultural sector).

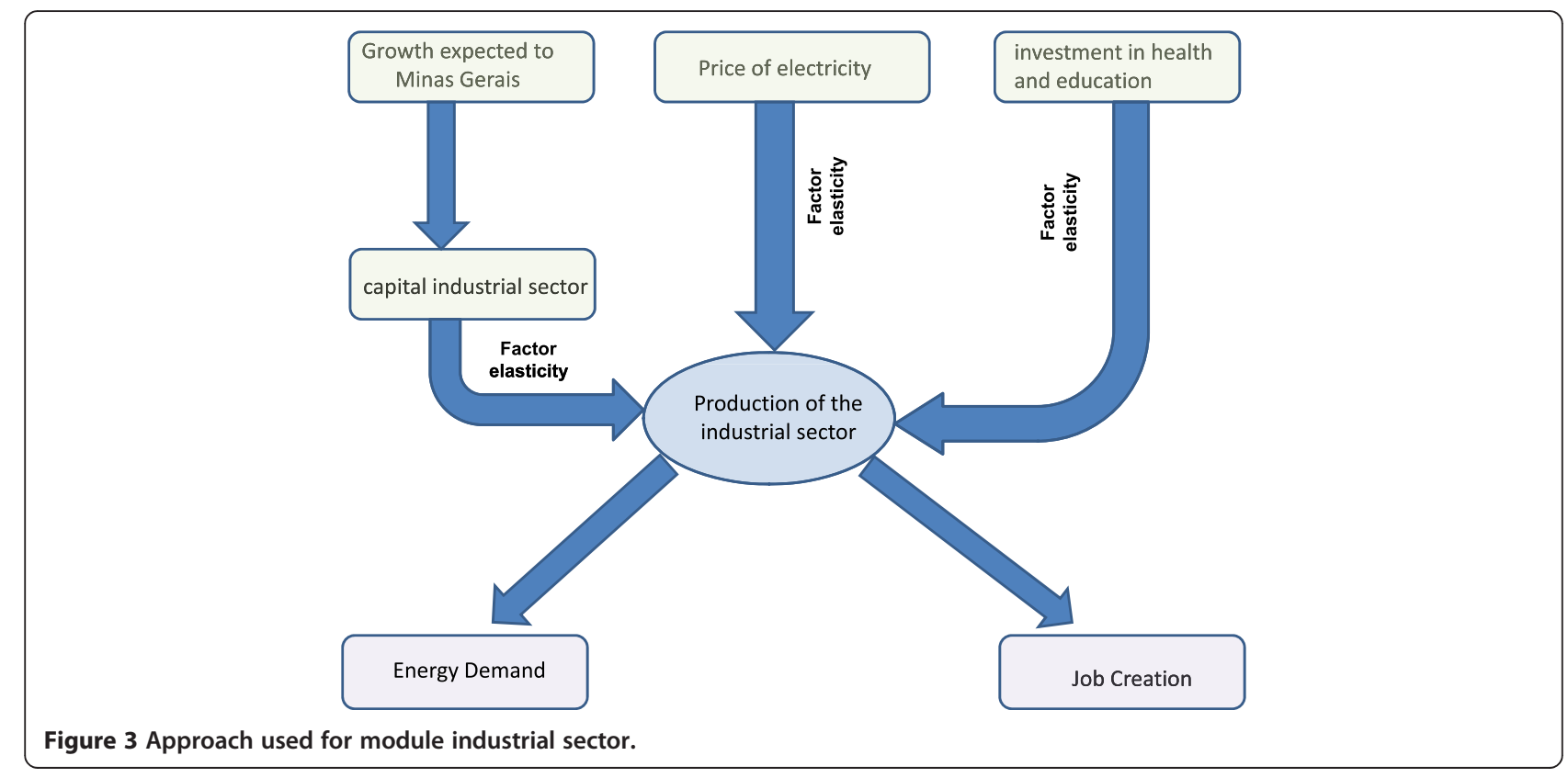


As a premise, the AFOLU sector is destined to be about $2 \%$ (about $12.000 \mathrm{~km}^{2}$ ) of the state territory for energy crops for ethanol and biodiesel. Thus, energy crops will not harm other crops.

The transportation sector module includes a variety of types of vehicles and fuels in the state. First, we projected the fleet for each type of vehicle (cars, motorcycles, vans, trucks, and buses) to 2030. The energy consumption of the fleet is based on assumptions about the share of biofuels, mainly ethanol and biodiesel, and increased energy efficiency for engines sold in Brazil. As a baseline assumption, the increasing efficiency of these engines is expected to maintain the trend observed in the recent years. In the BAU scenario, we considered an increase in the motors efficiency of $48 \%$ in 2050 relative to 2006, a constant share of ethanol and biodiesel in energy consumption, and no significant uptake of electric cars. In the RIC scenario, we assume that the governments of Brazil and Minas Gerais follow the US and the European laws in achieving lower fuel consumption (or higher fuel efficiency) by vehicle type [36]. We also assume that a government subsidy for ethanol will be established to ensure that it is competitive with gasoline and sugar. As a result, biodiesel penetration would increase by $2.5 \%$ every 5 years. Finally, we estimated the demand of fuel by type of vehicle and for the whole fleet. It is worth to note that due to the small share of natural gas in the energy mix in transport, we have not considered this fuel for this sector.

The electricity production sector is a key sector with respect to GHG emissions. For this sector, the primary driver is electricity demand (calculated previously in the residential, industrial, services, transport, and AFOLU sectors), allocated across various types of technology and energy sources, including a detailed representation of the capacity factor, capital and maintenance cost, employment, and emissions of each technology. The model then calculates the installed capacity for each source of energy, the cost of deployment, expected job creation in the energy sector, and its GHG emissions (including the carbon intensity of electricity imported). Due to the similarity of the electricity emission factor in Brazil and in the state of Minas Gerais, we have assumed that all the electricity imported will have the same carbon intensity as Minas Gerais.

Two scenarios were developed for the analysis of the results of the model, in the context of validation and policy analysis:

(1)BAU: We assumed that there are no major changes in the energy profile of the state (i.e., electricity supply is still dominated by hydroelectric generation, the transport matrix remains highly dependent on oil, and the vehicle fleet continues the exponential growth shown in the recent years). In this scenario, we did not consider additional public policies that would encourage the use of alternative energy sources, energy efficiency interventions, and the introduction of new technologies such as electric vehicles.

(2)RIC: This scenario represents an alternative development path assuming that a reduction in GHG emissions becomes a priority for the state government. For consistency, we assumed the same rates of economic growth and energy demand as in the BAU scenario; however, the differences in the energy mix and demand emerge as a result of the simulation of energy efficiency policies and the increase in the use of renewable energy. This scenario is also characterized by specific policies, such as the introduction of strong incentives for alternative energy sources (including financial subsidies), or the introduction of mandates for ethanol consumption, energy efficiency public policies and increased biodiesel content in diesel to $15 \%$ by 2030 . The scenario also considers the introduction of electric vehicles, reaching $50 \%$ of car sales in Minas Gerais in 2030 [37].

With the creation of scenarios up to 2030, it was also possible to estimate the costs of each intervention simulated. This was then coupled with the effectiveness in reducing GHG emissions and in creating new and additional jobs to carry out a more comprehensive policy analysis.

The validation of the model was carried out at the structural and behavioral level. The ultimate objective of the System Dynamics model validation is to establish the validity of the structure of the model. The accuracy of the model's reproduction of real behavior is also evaluated, but this is meaningful only if we first have sufficient confidence in the structure of the model. To begin with, direct structure tests assess the validity of the model structure by direct comparison with knowledge about the structure of the real system. This involves assessing each relationship within the model individually and comparing it with available knowledge about the real system. There is no simulation involved. Examples of direct structure tests include: (1) structure confirmation tests, (2) parameter confirmation tests, (3) direct extreme conditions test, and (4) dimensional consistency test (unit of measure check). Next, behavioral test are performed. Examples of behavior pattern tests include: (1) trend comparison and removal, (2) period comparison using the autocorrelation function, (3) comparison of the averages, (4) comparison of the variations, (5) testing for phase lag using the cross-correlation function, and (6) the computing the discrepancy coefficient. As an example, the results 
of the linear regressions analysis, using official data from other studies [35], indicate on average a correlation factor of approximately $95 \%$ for key variables in the model.

\section{Results and discussion}

The main results and indicators estimated for the two scenarios can be viewed in the comparative table below (Table 2).

To summarize the results, the RIC scenario has lower GHG emissions and greater energy generation and employment, but has a total cumulative cost of US\$34 billion (from 2013 to 2030), higher than BAU based on the estimated final indicators. On the other hand, the cumulative cost of 46 billion (resulted from oil derivates and electricity imports minus the electricity surplus exported) could be avoided, and the cumulative income generated through new employment could reach nearly US $\$ 4.4$ billion. The estimated employment refers to the additional direct job creation in the manufacturing, installation, operation, and maintenance of energy production capacity. As such, employment generation is proportional to production capacity, considering that different energy technologies have different labor requirements. In fact, the estimation of job creation is the result of the multiplication of construction and capacity by the specific employment multiplier of the technology and energy source considered. As such, the potential additional costs for employment (salary and wages) would be already incorporated in the generation cost and delivery of the respective technology and energy source.

Further, the energy demand shows a small decrease in the RIC scenario due to the energy efficiency policy implemented. The job creation in the energy sector was higher, explained by the alternative sources considered, like solar and wind, which are more labor intensive per

Table 2 Main results and final indicators of BAU and RIC scenarios

\begin{tabular}{ccc}
\hline Variables & BAU & RIC \\
\hline Installed power (MW) in 2030 & 21 thousand & 25 thousand \\
Energy production (MWh/year) in 2030 & 121 million & 131 million \\
Energy demand (MWh/year) in 2030 & 131 million & 127 million \\
Net import (MWh/year) & 10 million & -4 million \\
Oil demand (tonnes/year) in 2030 & 18 million & 14 million \\
Absolute $\mathrm{CO}_{2}$ equivalent emissions & 102 million & 92 million \\
(tonnes of $\mathrm{CO}_{2} \mathrm{e}$ ) & & \\
$\mathrm{CO}_{2}$ emission intensity (tonnes $\mathrm{CO}_{2} \mathrm{e} / \mathrm{MWh}$ ) & 0.84 & 0.70 \\
in 2030 & & \\
Energy sector employment (employer) & 10 thousand & 14 thousand \\
Avoided costs (US\$) & - & 46 billion \\
Additional income (US\$) & 2.8 billion & 4.4 billion \\
Total net cumulative cost (US\$) & 316 billion & 350 billion \\
\hline
\end{tabular}

MW installed. Below, we discuss the results of the main sectors analyzed.

\section{Energy demand}

In the BAU scenario, Minas Gerais would import electricity from 2026, as the expansion of supply cannot cope with the growth of demand (Figure 4).

The demand for electricity grows from around 56 million MWh/year to about 131 million MWh/year in 2030. This increase is due to the high GDP growth and increasing per capita consumption of electricity by the population, an approximate increase of $133 \%$ for electricity demand in the state. This result highlights the need to implement energy efficiency policies to mitigate the growth in demand or further expand supply (through imports in the BAU case). In the RIC scenario energy, the demand is lower due to energy efficiency interventions, such as the use of solar collector, extinction of incandescent bulbs, and increased efficiency in refrigerators, freezers, and air conditioners (Figure 4).

Additionally, the electricity production in the RIC scenario is much higher than the BAU scenario, through the encouragement of alternative energy sources and the re-powering of hydroelectric plants. Considering the introduction of alternative sources such as wind and solar, the state would still be energy self-sufficient by 2030 (Figure 4). Thus, the promotion of non-conventional energy sources, being alternative sources of energy for the reduction of GHG emissions, is also an excellent option for the state to maintain its energy security.

Oil demand, used for both transportation and electricity generation, shows strong growth in the BAU scenario (Figure 5). In 2030, oil demand is projected to reach about 18 million tonnes/year versus 10 million tonnes/year in 2010 , an increase of about $80 \%$, due in large part to growing demand from the vehicle fleet. In the RIC scenario, oil demand is mitigated to reach 14 million tonnes/year in 2030. This difference is justified mainly because of the greater use of ethanol in the transportation sector, the penetration of electric vehicles, and the substitution of thermal power generation for renewables.

The fuel demand for light vehicles refers to the demand for ethanol and gasoline in the state. Ethanol production also presents a strong growth in demand driven by the increasing number of automobiles and motorcycles in both scenarios. The demand for this fuel jumps from 1.4 billion liters to 4.6 billion liters in 2030 in the BAU scenario and to 6.8 billion liters in the RIC scenario. The difference between the two scenarios is due to public policies aiming at replacing gasoline with ethanol through economic subsidies.

Gasoline demand shows large differences for the two scenarios. While the BAU scenario considers the increase in the efficiency of internal combustion engines, 


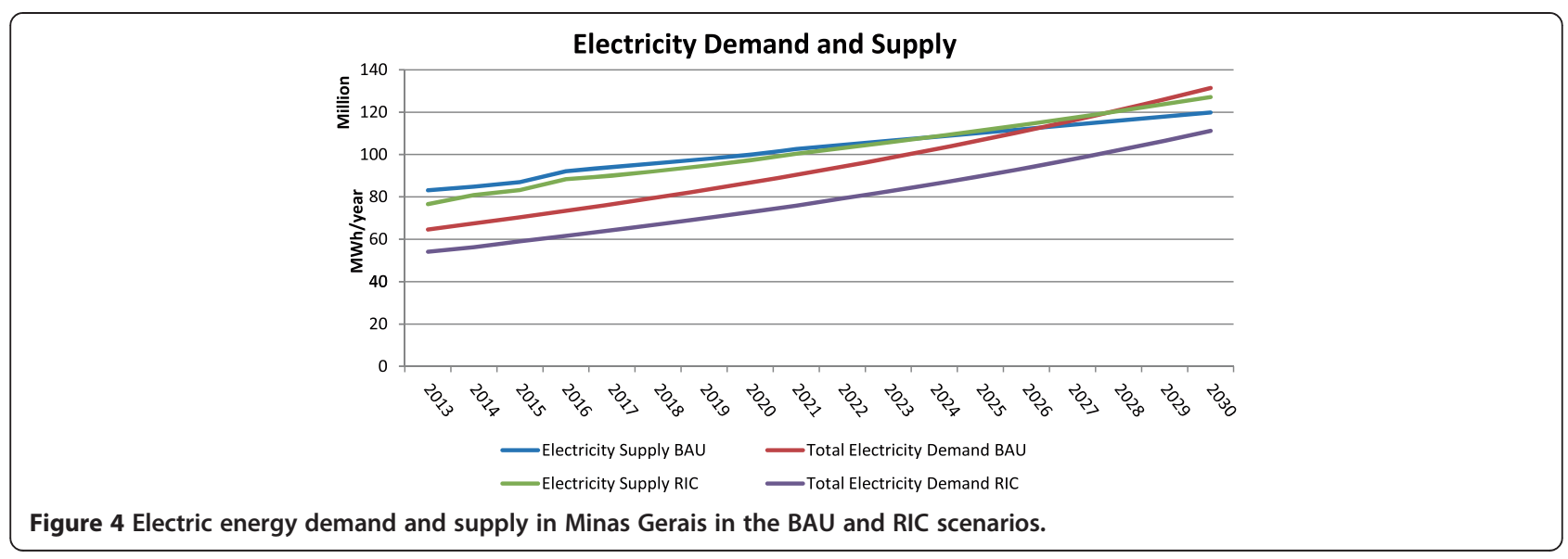

the RIC scenario adds the commercialization of electric vehicles and the implementation of an economic subsidy for ethanol, also supporting consumers and producers of sugarcane.

It is important to note that the full phaseout of gasoline through 2030 (as shown in Figure 6) is calculated primarily to estimate the potential achievable GHG reduction in the transport sector, rather than being a realistic government goal. However, we do not necessarily discard this scenario, especially considering the relevance of sugarcane production in the state (which is the third largest producer in the country) and the high potential to increase both productivity and production in the years to come. Nevertheless, this production has a cost, which is reflected in our calculations.

The fuel demand for heavy vehicles includes diesel and biodiesel consumption in the state (Figure 6). The demand for diesel in the RIC scenario shows a reduction of about 2 million tonnes/year due to the increased biodiesel blend in diesel and efficiency for diesel engines. We assumed a gradual increase of the biodiesel content in diesel through 2030, proportional to the increase in demand for diesel. Every 5 years, the assumed increase is $2.5 \%$ in the blend of biodiesel in diesel, resulting in jumps in demand for this fuel in the years 2015, 2020, 2025, and 2030. The BAU scenario does not provide for the increased biodiesel content in diesel, considering a low investment in the biodiesel production and market stagnation without the aid of public action. Comparing the demand and supply of natural gas observed in the RIC and BAU scenarios, we notice a slight increase due to the substitution of fuel oil and diesel with natural gas in power generation (Figure 7). Worth noting, the model did not consider the production of natural gas in Minas Gerais from the sedimentary basin of the São Francisco River due to the absence of robust technical and scientific data yet. However, unofficial sources estimate that the potential daily production is about 6 to $8 \mathrm{~m}^{3}$ [38], which would largely impact the energy mix of Minas Gerais by stimulating a significant increase in natural gas supply. If estimates are confirmed and the potential is actually exploited, we can expect a drop in natural gas prices and the replacement of LPG gas in the residential sector.

\section{Power generation}

In both scenarios, we considered only the construction of plants already approved, since the potential capacity is already close to saturation, and environmental legislation

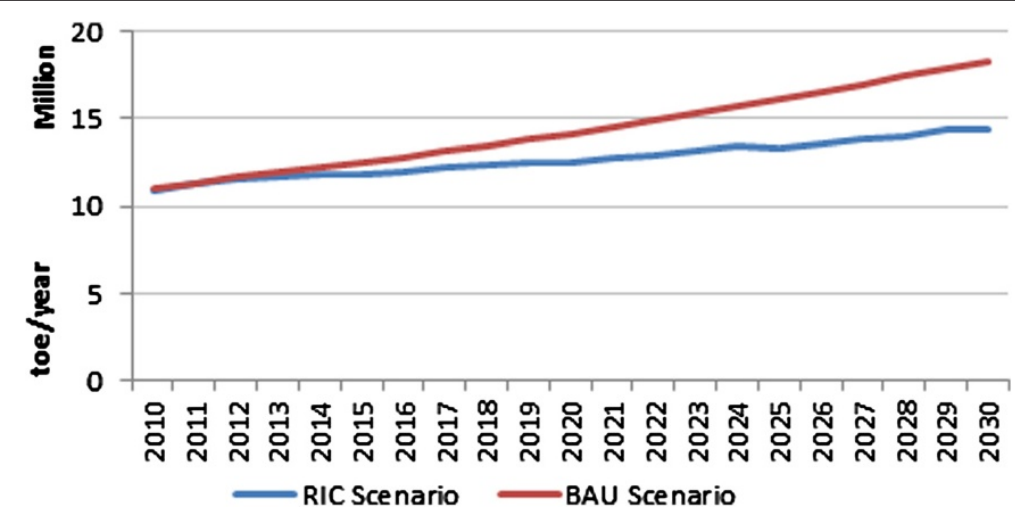

Figure 5 Total oil demand in Minas Gerais for BAU and the RIC scenarios. 


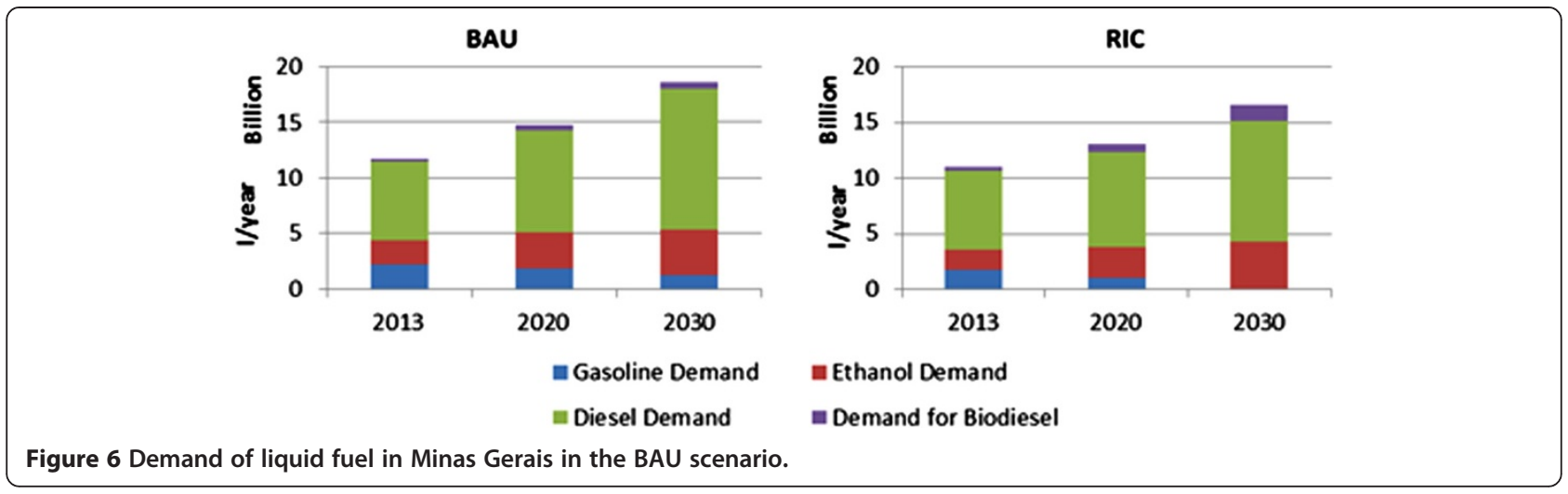

is increasingly resistant to the construction of larger dams [4]. The installed capacity in the RIC scenario shows an increase of 5\% compared to the BAU scenario. The difference between the two scenarios results from the refurbishment and improvement of old plants with higher efficiency technology, adding $20 \%$ to the initial power capacity of the plant.

In the case of wind power, while the BAU scenario foresees an annual increase of $74 \mathrm{MW}$ of installed power, the low-carbon scenario is more daring and provides an exponential increase until the state reaches $10 \%$ of its potential wind capacity in 2030 (about 3,900 MW) (Figure 8).

The installed capacity of solar photovoltaic showed the greatest discrepancy between the two scenarios. While the BAU scenario was set up to be very conservative to reach about $4.5 \mathrm{MW}$ by 2030 , the RIC scenario has the same growth achieved by Germany (nearly $30 \%$ per year) and achieve the mark of $250 \mathrm{MW}$ in the state by 2030 (Figure 8).

\section{Greenhouse gas emissions}

Finally, when comparing the two emissions scenarios, GHG emissions will grow in both scenarios until the year 2030 as shown in Figure 9. However, the increase in the RIC scenario occurs in spite of greater electricity generation compared to the BAU scenario. Thus, the carbon intensity of electricity indicator (the ratio of GHG emissions and electricity generated) reaches a value of 0.7 tonnes of $\mathrm{CO}_{2} \mathrm{e} / \mathrm{MWh}$ in the RIC scenario as opposed to 0.84 tonnes of $\mathrm{CO}_{2} \mathrm{e} / \mathrm{MWh}$ in the $\mathrm{BAU}$ scenario as shown in Figure 10.

Compared to 2010 (0.82 tonnes of $\mathrm{CO}_{2} \mathrm{e} / \mathrm{MWh}$ ), the RIC scenario would reach a reduction in carbon intensity for power generation of about $15 \%$. The same does not occur in the BAU scenario, which actually shows an increase in carbon intensity.

If we consider the carbon intensity of all energy consumed, we have a decrease of $16 \%$ due to the difference of 0.84 and 0.70 tonnes of $\mathrm{CO}_{2} \mathrm{e} / \mathrm{MWh}$ consumed in the BAU and RIC scenarios, respectively.

\section{Cost of implementing the BAU and RIC scenarios}

We estimated the annual and cumulative cost for achieving the energy supply required in both scenarios. This would be the total amount of funding that the public and/or private sector, depending on the interventions implemented, will have to disburse to reach the desired levels of energy demand and supply simulated in the RIC scenario (Table 3). In fact, the costs of the scenarios represent the overall energy bill, considering both capital investment and trade, as the sum of all the investments

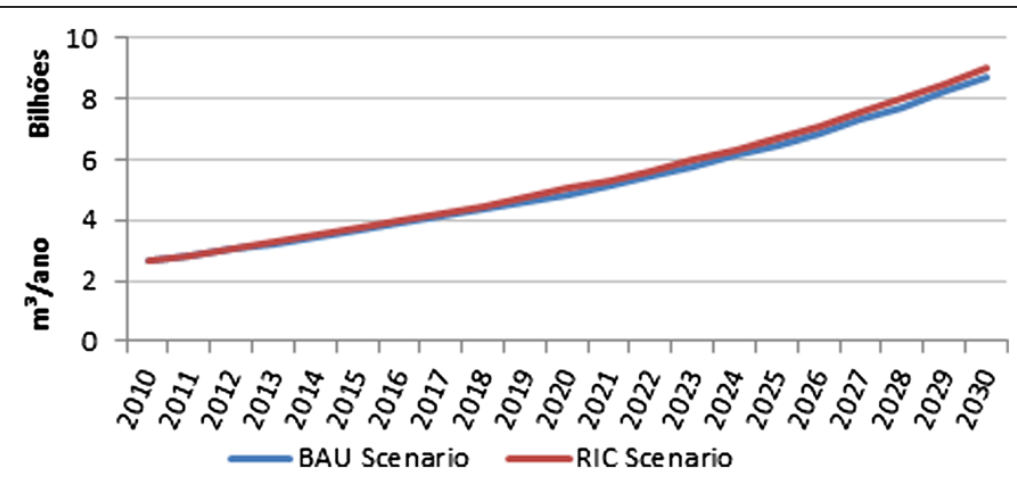

Figure 7 Demand of natural gas in Minas Gerais in the BAU and RIC scenarios. 


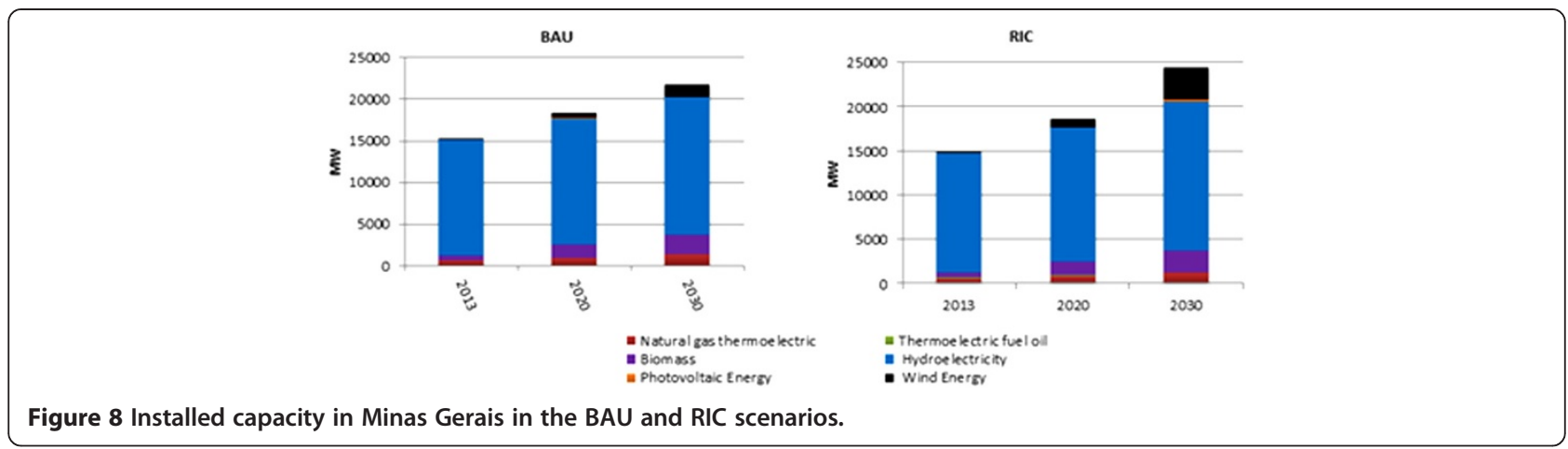

needed to expand the capacity of power plants, the cost of energy imports (fossil fuels and electricity), oil refining, and internal electricity production for each energy source analyzed.

In the BAU scenario, we estimate that the average annual cost and total cumulative cost of all interventions reach US $\$ 18.5$ billion per year and US $\$ 316$ billion, respectively. Initial estimates indicate that this scenario would generate (and require) approximately 10,000 direct jobs in the energy sector. In addition, the annual cost of electricity imports by 2030 could reach US $\$ 449$ million.

In the RIC scenario instead, the average annual cost and the total cumulative cost were estimated to be approximately US $\$ 21$ billion per year and US $\$ 350$ billion, respectively. The additional investment required is the result of the expansion of capacity for power generation from alternative sources (e.g., wind and solar) that are currently more expensive than hydroelectric plants. Of relevance, the investment estimated also includes the public subsidy (financial) for ethanol production, supporting producers by equating their sale price to the price of international sugar. The initial estimates indicate that this scenario would generate (and require) approximately 14,000 direct jobs only in the energy sector.

Besides the benefits of energy security (with the state being still energy self-sufficient in the RIC scenario until
2030 and beyond), the results show an important cumulative economic savings of roughly US $\$ 1$ billion by 2030 due to avoided electricity imports and increased electricity exports.

In addition, we calculated the income generated by increasing direct employment in the energy sector. The BAU scenario is projected to generate US $\$ 301$ million/ year, which grows to US\$536 million/year in 2030 in the RIC scenario. This difference in income generation was estimated to impact the state GDP by increasing growth by at least $0.07 \% /$ year. Since we calculated only the direct new jobs in the energy sector, the GDP growth mentioned above can be considered conservative, because the model does not capture the indirect effects of investments into "greening" the economy, resulting in additional indirect jobs, increased average wages, and higher consumption.

\section{Conclusions}

In light of the results presented in the paper, we can appreciate the magnitude of the challenge that the state will need to address over the coming years to mitigate GHG emissions and provide energy security in the state. The estimated investments needed to achieve energy security and low carbon intensity in the RIC scenario represent an average increase of US $\$ 2$ billion annually over the BAU case, which represents $0.57 \%$ of the state GDP in 2010.

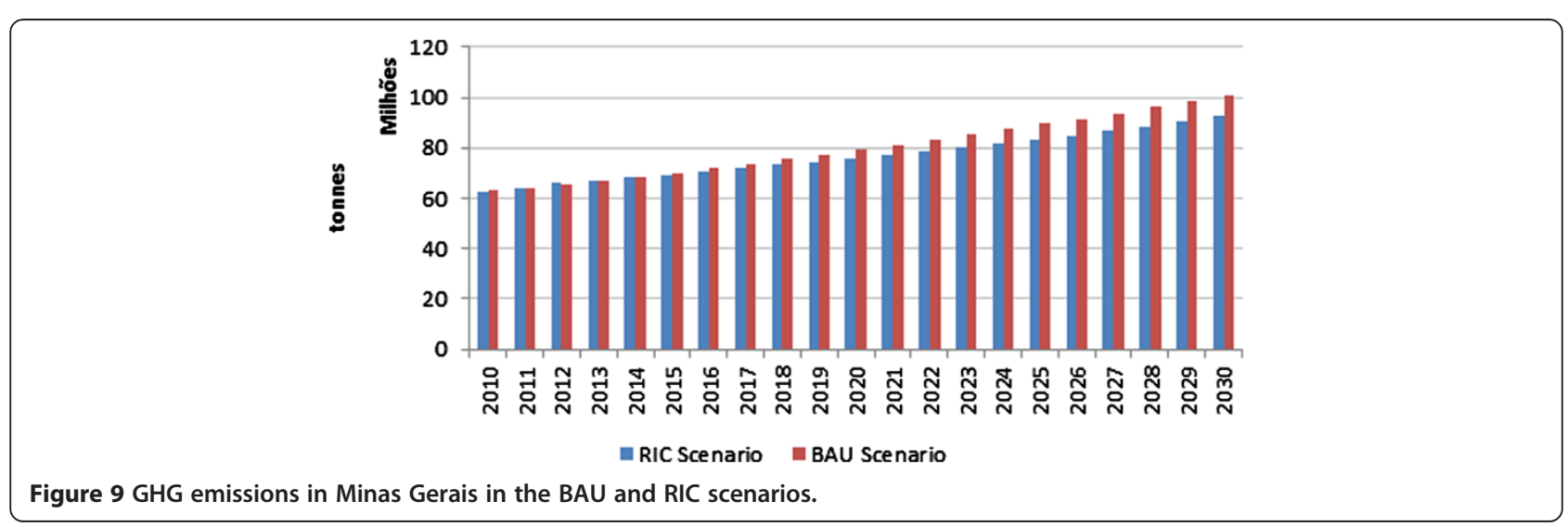




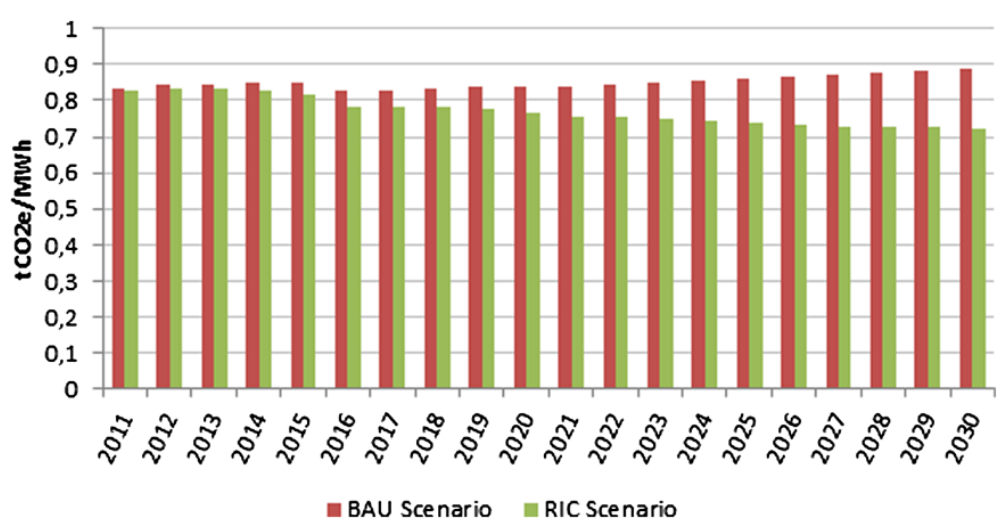

Figure 10 Carbon intensity of electricity in the BAU and RIC scenarios.

On the other hand, we can see important opportunities to contribute to global climate change mitigation while fostering a low-carbon energy sector and a green economy in the state. By investing in the renewable energy sources, energy efficiency, technological improvements, and the targets analyzed in this study, Minas Gerais State could reduce political and financial risks originating from the reliance on fossil fuels while generating more income in the energy sector and the state economy as a whole and reducing medium- and longerterm costs.

Table 3 Estimated costs of interventions by 2015 and 2030, and cumulative costs in BAU and RIC scenarios

\begin{tabular}{|c|c|c|c|c|c|c|}
\hline \multirow{2}{*}{$\begin{array}{l}\text { Estimated costs } \\
\text { (in US\$ million) }\end{array}$} & \multicolumn{2}{|c|}{2015} & \multicolumn{2}{|c|}{2030} & \multicolumn{2}{|c|}{ Cumulative } \\
\hline & BAU & RIC & BAU & RIC & BAU & RIC \\
\hline Power generation & 455 & 426 & 789 & 847 & 7,597 & 8,859 \\
\hline $\begin{array}{l}\text { Imports of oil } \\
\text { derivates }\end{array}$ & 2,287 & 1,454 & 9,249 & 4,346 & 54,934 & 24,946 \\
\hline Oil production & 10,025 & 9,992 & 10,577 & 10,787 & 135,590 & 135,590 \\
\hline $\begin{array}{l}\text { Construction of } \\
\text { renewable power } \\
\text { plants }\end{array}$ & 2,454 & 1,823 & 462 & 939 & 8,497 & 10,862 \\
\hline $\begin{array}{l}\text { Construction of } \\
\text { non-renewable } \\
\text { power plants }\end{array}$ & 117 & 41 & 469 & 67 & 3,192 & 1,245 \\
\hline Electricity exports & $-1,194$ & $-1,332$ & 0 & -471 & $-14,892$ & $-30,391$ \\
\hline Electricity imports & 0 & 0 & 0.449 & 0 & 0.590 & 0 \\
\hline Oil imports & 5,726 & 5,726 & 8,798 & 8,798 & 121,083 & 121,083 \\
\hline $\begin{array}{l}\text { Economic subsidy } \\
\text { for ethanol } \\
\text { production }\end{array}$ & 0 & 0 & 0 & $7,746^{\mathrm{a}}$ & 0 & $78,256^{a}$ \\
\hline $\begin{array}{l}\text { Income generated } \\
\text { from job creation }\end{array}$ & 34 & 86 & 301 & 536 & 2,800 & 4,400 \\
\hline Total net cost & 19,872 & 18,132 & 31,494 & 33,061 & $316,143^{b}$ & $350,452^{b}$ \\
\hline
\end{tabular}

${ }^{a}$ Calculated to keep the same payment for the producer, considering sugar or alcohol yield. This high cost is due to the price of sugar rises from US\$139 to US\$210/t (in 2030), while the price of alcohol (endogenous) suffers slight variation from US\$298/t to US\$300/t.

${ }^{\mathrm{b}}$ Cumulative costs estimated from 2013 to 2030.
Further, it is crucial to note that investments in research and development in the energy sector are critical to ensure that the interventions simulated can be technically and economically viable. Another point to be considered is the need to review the transportation model in the state, which is focused primarily on road transport (single mode). Large part of the total GHG emissions refers to fossil fuels used for all logistics of people and goods in the state.

In addition, we intend to refine the model adding new modules, such as urban mobility/intermodal and fleet growth, exploration/production of natural gas in Minas Gerais, and power generation through waste. Additional sectors to be added include a more detailed representation of society and the economy, adding details and exploring additional cross-sectoral feedback loops with the goal to more explicitly support policy formulation and evaluation.

Despite the intrinsic uncertainties characterizing every modeling approach, this study highlights the importance of medium- and long-term planning in the energy sector, naturally carried out by the government due to its economically strategic and social relevance, in order to utilize advanced methods and tools that can be used to explore possible energy scenarios and their implications for public policies. Also, our results confirm and strengthen the role of investments in ensuring energy security while lowering GHG emissions, as a valid public policy options to help "greening" the energy sector in a more sustainable national and subnational context.

\section{Abbreviations}

AFOLU: Agriculture, forestry, and other land uses; BAU: Business as usual; CGE: Computable general equilibrium; $\mathrm{CO}_{2}$ eq: Equivalent carbon dioxide; ETL: Endogenous technology learning; GDP: Gross domestic product; GEM: General equilibrium model; GHG: Greenhouse gas; MAED: Model for analysis of energy demand; MARKAL: Market allocation; MED: Macro (non-linear) and elastic demand; MESSAGE: Model of energy supply systems alternatives and their general environmental impacts; RIC: Reduction of carbon intensity; T21: Threshold 21; WEM: World energy model. 


\section{Competing interests}

The authors declare that they have no competing interests.

\section{Authors' contributions}

MX developed the platform modules, carried out the calibration and validation of the model, and drafted the manuscript. AB provided guidance to the team in the development of the model and its structural and behavioral validation. $A B$ also carried out the literature review and contributed to the preparation of the manuscript. KS participated in designing the reference scenario. WPBF added the scenarios of wind power and its consistency. CMdS revised the language and the concepts presented in the first manuscript. FN conceived of the study and participated in its design and coordination. FN also contributed to the preparation of the manuscript. All authors read and approved the final manuscript.

\section{Acknowledgements}

This work was supported by Fundação de Amparo à Pesquisa do Estado de Minas Gerais (FAPEMIG).

\section{Author details}

${ }^{1}$ Fundação Estadual do Meio Ambiente (FEAM), Rodovia Prefeito Américo Gianetti, s/n, Belo Horizonte, Minas Gerais 31630-900, Brazil. ${ }^{2}$ KnowlEdge Srl, 35, via Col Di Lana, Castellanza 21053, Italy. ${ }^{3}$ Sustainable Development Programme of the School of Public Leadership, Faculty of Economic and Management Sciences, Stellenbosch University, Private Bag X1, Stellenbosch 7602, South Africa.

\section{Received: 29 May 2013 Accepted: 7 August 2013}

Published: 28 August 2013

\section{References}

1. United Nations Environment Programme (2011) Towards a green economy: pathways to sustainable development and poverty eradication., http://www. unep.org/greeneconomy/Portals/88/documents/ger/GER_synthesis_en.pdf. Accessed 04 Sept 2012

2. United States Energy Information Administration (2011) Annual energy outlook 2011. United States Energy Information Administration. US Department of Energy, Washington

3. APREN (2009) Deloitte consultores. Associação de Energias Renováveis, Lisboa

4. Companhia Energetica de Minas Gerais (2011) 25 Balanço energético do estado de Minas Gerais. Companhia Energetica de Minas Gerais, Belo Horizonte

5. Fundação Estadual do Meio Ambiente (2008) Inventário de emissões de gases de efeito estufa do estado de Minas Gerais. Fundação Estadual do Meio Ambiente, Belo Horizonte

6. Companhia Energetica de Minas Gerais (2010) $24^{\circ}$ balanço energético do estado de Minas Gerais. Companhia Energetica de Minas Gerais, Belo Horizonte

7. Bunn DW, Larsen ER (eds) (1997) Systems modelling for energy policy. Wiley, Chichester

8. Sterman JD (2000) Business dynamics: systems thinking and modeling for a complex world. Irwin/McGraw-Hill, Boston

9. Bassi AM (2009) An integrated approach to support energy policy formulation and evaluation. Department of Geography, System Dynamics Group, University of Bergen, Norway

10. Martinsen D, Krey V (2008) Compromises in energy policy—using fuzzy optimization in an energy systems model. Energy Policy 36(8):2983-2994

11. Loulou R, Goldstein G, Noble K (2004) Documentation for the MARKAL family of models. IEA Energy Technology Systems Analysis. Programme, Paris

12. Messner S, Schrattenholzer L (2000) MESSAGE-MACRO: linking an energy supply model with a macroeconomic model and solving it interactively. Energy 25:267-282

13. Fishbone LG, Giesen G, Goldstein G, Hymmen HA, Stocks KJ, Vos H, Wilde D, Zöcher R, Balzer C, Abilock H (1983) User's guide for MARKAL. IEA Energy Technology Systems Analysis. Programme, Paris

14. Messner S, Golodnikov A, Gritsevskii A (1996) A stochastic version of the dynamic linear programming model MESSAGE III. Energy 21:775-784

15. Messner S, Strubegger M (1995) User's guide for MESSAGE III. International Institute for Applied Systems Analysis. Laxenburg, Austria

16. International Energy Agency (2004) World energy outlook 2004, annex C: world energy model. International Energy Agency, Paris
17. Energy Information Administration, US Department of Energy (2003) The national energy modeling system: an overview. Energy Information Administration, Washington, DC

18. International Energy Agency (2006) World energy model - methodology and assumptions. In: World energy outlook 2006. OECD Publishing, Paris

19. International Institute for Applied Systems Analysis (2001) Model MESSAGE, command line user manual, version 0.18. IIASA, Laxenburg

20. International Institute for Applied Systems Analysis (2002) Achieving a sustainable energy system. IIASA, Laxenburg

21. Institute of Computers and Communications Systems (2006) GEM-E3 model manual. National Technical University of Athens, Greece

22. Drud A, Grais W, Pyatt G (1986) Macroeconomic modeling based on social accounting principles. J Policy Model 8(1):111-145

23. Bassi AM (2010) Evaluating the use of an integrated approach to support energy and climate policy formulation and evaluation. Energies 3(9):1604-1621. doi:10.3390/en3091604

24. AES Corporation (1993) An overview of the IDEAS Model: a dynamic long-term policy simulation model of US energy supply and demand. AES Corporation, Arlington

25. Naill RF (1977) Managing the energy transition. Ballinger, Cambridge, MA

26. Backus G, Green J, Masevice A (1979) FOSSIL 79: documentation. Resource Policy Center, Dartmouth College, Hanover

27. Sterman JD (1981) The energy transition and the economy: a system dynamics approach. Dissertation, Sloan School of Management, MIT, Cambridge, MA

28. Davidsen PI, Sterman JD, Richardson GP (1990) A Petroleum Life Cycle Model for the United States with endogenous technology, exploration, recovery, and demand. Syst Dynam Rev 6(1):66-93

29. Fiddaman TS (1997) Feedback complexity in integrated climate-economy models. Sloan School of Management, MIT, Cambridge, MA

30. Bassi AM, Shilling JD (2010) Informing the US energy policy debate with Threshold 21. Technol Forecast Soc 77:396-410

31. Bassi AM, Schoenberg W, Powers R (2010) An integrated approach to energy prospects for North America and the rest of the world. Energ Econ 32:30-42. doi:10.1016/j.eneco.2009.04.005

32. Loulou R, Remme U, Kanudia A, Lehtila A, Goldstein G (2005) Documentation for the TIMES Model. IEA Energy Technology Systems Analysis. Programme, Paris

33. Dutch National Research Programme on Global Air Pollution and Climate Change (2002) Uncertainty Assessment of the IMAGE/TIMER B1 $\mathrm{CO}_{2}$ emissions scenario, using the NUSAP Method. Dutch National Research Programme on Global Air Pollution and Climate Change, Netherlands

34. Millennium Institute (2012) Threshold 21 (T21) North America Model and user guide. Millennium Institute, Arlington

35. Universidade Federal do Rio de Janeiro (2007) Matriz energética de Minas Gerais 2007-2030. Universidade Federal do Rio de Janeiro, Brazi

36. Weiss D, Weidman J (2012) How big oil spent part of its $\$ 90$ billion in profits so far in 2012. Center American for Progress, Washington, DC

37. Paixão PDTG (2012) World energy insight 2010. World Energy Council, São Paulo

38. Vieira M (2012) Produção de gás natural em Minas Gerais começa este ano. EM Jornal Estado de Minas, Belo Horizonte

doi:10.1186/2192-0567-3-17

Cite this article as: Xavier et al:: Energy scenarios for the Minas Gerais State in Brazil: an integrated modeling exercise using System Dynamics. Energy, Sustainability and Society 2013 3:17. 